\title{
Acanthamoeba keratitis: confirmation of the UK outbreak and a prospective case-control study identifying contributing risk factors
}

\author{
Nicole Carnt, 1,2,3 Jeremy J Hoffman, ${ }^{3}$ Seema Verma, ${ }^{3}$ Scott Hau, ${ }^{3}$ Cherry F Radford, ${ }^{3}$ \\ Darwin C Minassian, ${ }^{4}$ John K G Dart ${ }^{3,5}$
}

\begin{abstract}
- Additional material is published online only. To view please visit the journal online (http://dx.doi.org/10.1136/ bjophthalmol-2018-312544).
\end{abstract}

${ }^{1}$ School of Optometry and Vision Science, University of New South Wales, Sydney, New South Wales, Australia

${ }^{2}$ Centre for Vision Research, Westmead Institute for Medical Research, Westmead, Australia ${ }^{3}$ Corneal and External Disease Service, Moorfields Eye Hospital NHS Foundation Trust, London, UK

${ }^{4}$ EpiVision Ophthalmic Epidemiology Consultants,

Penn, UK

${ }^{5}$ National Institute of Health Research (NIHR) Biomedical Research Centre at Moorfields Eye Hospital NHS Foundation Trust and UCL Institute of Ophthalmology, London, UK

Correspondence to Professor John K G Dart, Moorfields Eye Hospital, London EC1V 2PD, UK; J.Dart@ucl.ac.uk

Received 12 May 2018 Revised 27 July 2018 Accepted 7 August 2018 Published Online First 19 September 2018

\begin{abstract}
Background/aims Acanthamoeba keratitis (AK) is a chronic debilitating corneal infection principally affecting contact lens $(\mathrm{CL})$ users. Studies were designed to test claims that the UK incidence may have increased in 2012-2014 and to evaluate potential causes.

Methods Annualised incidence data were collected from January 1984 to December 2016. Case-control study subjects were recruited between 14 April 2011 and 05 June 2017. Reusable CL users with AK were recruited retrospectively and prospectively. Controls were reusable $\mathrm{CL}$ users, recruited prospectively, with any disorder other than AK. Multivariable analysis of questionnaire data measured independent risk factors for AK.

Results The current outbreak of AK started in 2010-2011 with an incidence threefold higher than in 2004-2009. Risk factors for AK were: Oxipol disinfection, CLs made of group IV CL materials, poor CL hygiene, deficient hand hygiene, use of CLs while swimming or bathing, being white British, and for those in social classes 4-9.

Conclusion AK is a largely preventable disease. The current outbreak is unlikely to be due to any one of the identified risk factors in isolation. Improving $\mathrm{CL}$ and hand hygiene, avoiding CLs contamination with water and use of effective $C L$ disinfection solutions, or daily disposable $\mathrm{CLS}$, will reduce the incidence of AK. In the longer-term, water avoidance publicity for $\mathrm{CL}$ users can be expected to reduce the incidence further. Ongoing surveillance of AK numbers will identify changes in incidence earlier. Evaluation of Acanthamoeba contamination in end-user drinking water would contribute to our understanding of regional variations in the risk of exposure.
\end{abstract}

\section{INTRODUCTION}

Acanthamoeba spp are free-living cyst-forming protozoans, ubiquitous in air, soil, dust and water, to which $50 \%-100 \%$ of us develop antibodies. However, infections are uncommon, rarely involving the brain but more often the cornea. Acanthamoeba exist as a vegetative trophozoite, usually feeding on other microorganisms, whereas in the cornea, they probably feed on keratocytes. In adverse environments, including the nutrient deficiency and noxious treatments that the organisms are exposed to in keratitis, trophozoites encyst. The cysts are extremely resilient and are the form of the organism responsible for persistent relapsing keratitis. $^{1}$
Despite its comparative rarity, and status as an orphan disease, Acanthamoeba keratitis (AK) is of concern because of its severe and prolonged morbidity in the young and economically active contact lens (CL) users who constitute 90\% of affected patients in the UK. The most severely affected quartile require more than: 10 months treatment, 38 months follow-up, 31 hospital visits, have less than 6/24 vision after resolution and require corneal transplants. ${ }^{2}$ Established independent risk factors for developing the disease have been exposure to water; in the context of CL use, this risk has been related to exposure to domestic tap water both in the home, ${ }^{34}$ and while swimming or bathing when wearing CLs. ${ }^{45}$ Others have been poor CL hygiene, ${ }^{4-6}$ orthokeratology use of rigid $\mathrm{CLs}^{6}$ and lens disinfection solution failures which have resulted in previous outbreaks of AK both in the UK and USA. ${ }^{78}$ Following the last UK outbreak in 1991-1995, a national incidence study reported a mean of 53 cases per annum (p.a.) of which $88 \%$ were in CL users ${ }^{4}$; less than half the annual incidence at the peak of that outbreak. ${ }^{7}$

Since then, no increase in annual AK incidence has been noticed until concerns expressed in 2012 2014. ${ }^{910}$ As a result, we initiated the two studies reported here. Our results are likely to have relevance to the whole UK given that in 1997-1999, Moorfields Eye Hospital (Moorfields) treated $>75 \%$ of AK cases in the south east and $>35 \%$ of all UK cases. ${ }^{4}$ The incidence study was to measure the annualised incidence at Moorfields. The case-control study was designed to identify potential causes of AK, which we hypothesised might be largely due to ineffective CL solutions given the findings from previous outbreaks. $^{711}$

\section{METHODS}

Incidence study of AK at Moorfields 1984-2016

The data collection methodology for the numbers of cases of AK cases seen at Moorfields from January 1984 to December 2016 has differed for different periods and are not directly comparable. The methodology is described in figure $1 .{ }^{471213}$

\section{Case-control study}

Patients using CLs and attending Moorfields Accident and Emergency (A\&E) Department completed a self-administered questionnaire based on those used in previous studies, ${ }^{7} 14$ providing data on demographics, lens type, wear schedule, lens care 


\section{Acanthamoeba keratitis cases at Moorfields 1984-2016}

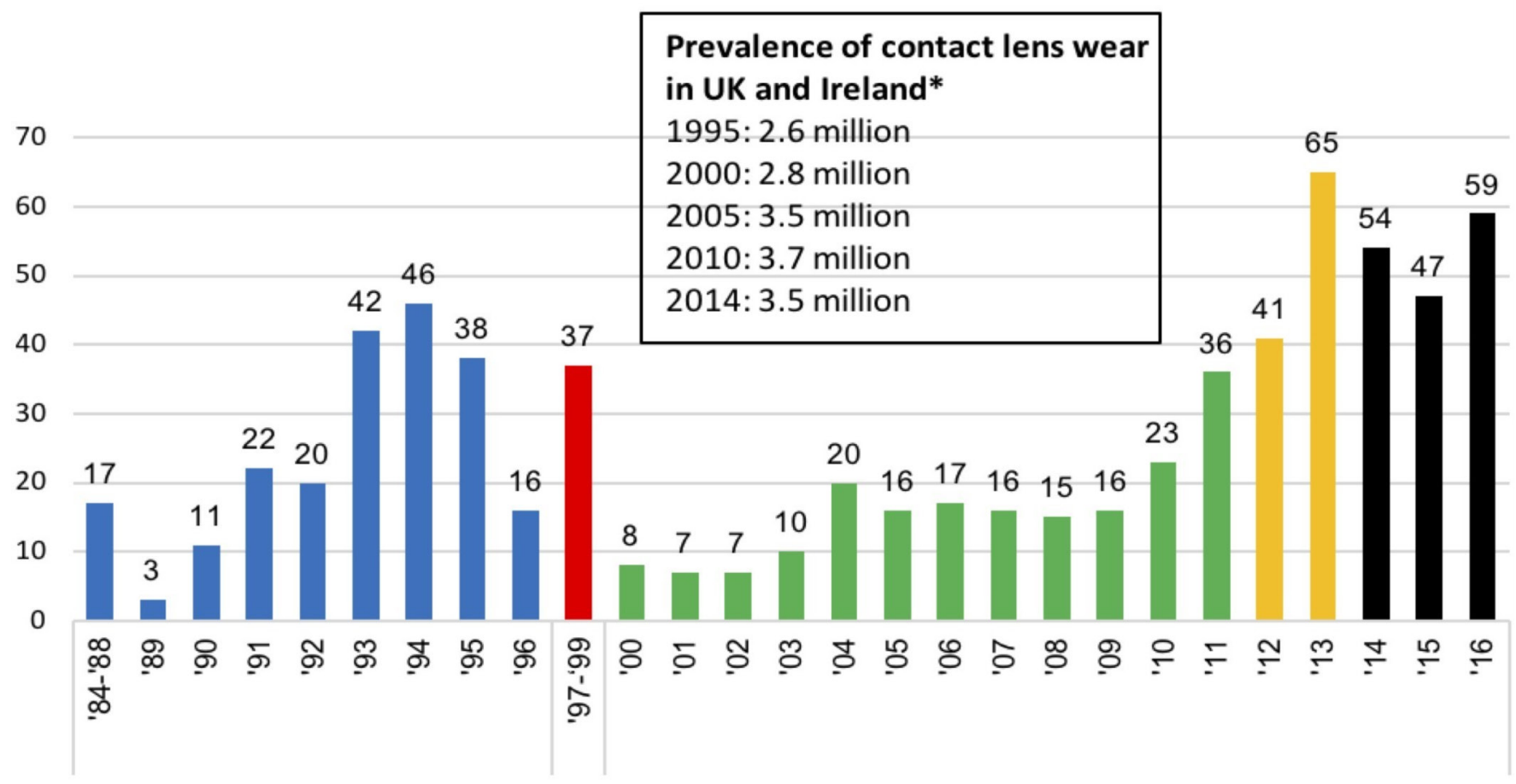

Figure 1 The total number of cases from 1984 to 2016 (33 years) totalled 709. The data collection methodology varied for different periods labelled (A)-(E) in the text below and by colour in the figure. The colours identify the periods for which the same data collection methodology was used. The references retain the numbering they have in the text: (A) 1984-1996 data (blue bars) were published in a letter as a bar chart ${ }^{12}$ and was collected using the clinical and/or microbiological (culture or histology) criteria used in a previous study. ${ }^{7}$ (B) 1997-1999 data (red bar) were available from a national survey ${ }^{4}$ for a 24-month period 01 October 1997 to 30 September 1999 for which annual figures are not available: there were 37 cases in this 2-year period. (C) From 2000 to April 2012 (green bars) cases were identified from our current microbiology laboratory electronic database, and an electronic letter search of our electronic patient database, both of which started in 2000. Criteria for inclusion were a positive Acanthamoeba culture, histopathological confirmation of trophozoites and/or cysts, culture-negative cases shown to have Acanthamoeba cysts on confocal microscopy, and those with a typical clinical course and response to treatment. ${ }^{4}$ (D) From March 2012 to December 2013 (yellow bars) cases were identified prospectively as part of studies being carried out on Acanthamoeba keratitis (AK) using the criteria described for the (C) period. (E) From 01 January 2014 to 31 December 2016 (black bars) cases were identified by retrospective audit, using the same criteria as for (C) but with the addition of Acanthamoeba DNA identification by PCR as an additional inclusion criterion. " ${ }^{* \prime}$ represents https://www.statista.com/statistics/429790/wearers-ofcontact-lenses-united-kingdom-ireland/ (data from the Association of Contact Lens Manufacturers market report 2014: technical summary).

and frequency of disposal, lens wear experience, frequency of practitioner aftercare, showering and bathing when wearing CLs and smoking.

Cases were reusable daily wear CL users diagnosed with AK having an initial attendance in the Moorfields A\&E service. These included both self-referrals, secondary (general practitioner and optometric) and tertiary (other ophthalmology centres) referrals. These were identified between 14 April 2011 and 28 August 2014. Cases diagnosed before ethics approval was given on 18 February 2013 were recruited after diagnosis, subsequent to ethics approval cases were recruited at the time of diagnosis. Inclusion criteria for AK cases were the same as those used for the incidence study and are described in figure 1C,E.

Controls were recruited prospectively. Like the cases, these were attending Moorfields A\&E. Inclusion criteria were daily wear CL wearers using CL solutions as part of the hygiene routine for reusable CL's having any disorder other than AK. Controls were identified in two separate periods. This definition excluded users of true daily disposable and overnight (also known as continuous or extended) wear soft lenses for which no CL solutions were used. A first set of controls was collected between
17 February 2014 and 11 June 2015 and a second between 22 November 2016 and 05 June 2017. A diagnosis for each questionnaire respondent was derived from the hospital notes. Disorders in the control CL user populations were classified into two subsets: those with CL-related diseases and those with diseases thought to be unrelated to CL wear. These conditions are listed in online supplementary table 1). CL-related diseases included all CL-associated keratitis, other than AK, defined using criteria from previous studies. ${ }^{14-16}$

CL solutions were classified and analysed by the principal active ingredient using information derived from the packaging or manufacturers where possible or retailers when we could not establish the formulation from the first two sources. The classification is in online supplementary table 2 .

CLs were classified and analysed by their material using a simplification of the American National Standards Institute Terminology for Contact Lenses Reference Z80.20-2016 and checked for each CL brand, or rebranded lens, against the UK Association of Contact Lens Manufacturers Annual Handbook for the years of the study. These data are summarised in online supplementary table 3. 


\section{Statistical methods}

The main analysis sample included all eligible controls collected during 2016-2017. A sample size of 60 cases and 180 controls was expected to provide $85 \%$ power (alpha 0.05) to detect a minimum odds ratio (OR) of $2.7 \%$ assuming $20 \%$ of the controls were exposed. Initial assessment of potential risk factors was carried out one at a time using logistic regression without adjustment for confounding and is described in online supplementary table 4. CL hygiene compliance is an important potential risk factor and the methodology for calculating the score used for this analysis is described in online supplementary table 5. Following this initial assessment, multiple logistic regression models were constructed to obtain estimates of ORs adjusted for confounding effects. ORs are reported in this study as estimates of relative risk. Variables selected initially for inclusion in the model building process were those having $\mathrm{p}$ values of $<0.2$ from the unadjusted analysis together with variables thought a priori to be risk factors for AK: hygiene score, hand washing before lens handling and the principal active ingredient of the disinfection solution. The distribution of cases and controls for these variables are shown in table 1 . In constructing each final multivariate (MV) model, one exposure variable was considered as the 'exposure of main interest' and all others as 'auxiliary factors' (potential confounders). The final MV model was thus optimised to estimate the adjusted OR for the 'exposure of main interest'; covariate adjustment is described in online supplementary table 6 . The performance of logistic modelsgoodness-of-fit and discriminatory ability-were assessed by calculating the Hosmer-Lemeshow $\mathrm{p}$ value and area under the receiver operating characterisitic (ROC) curve, respectively. The statistical package used was Stata V.14.2 (StataCorp).

\section{RESULTS \\ Incidence study}

Figure 1 shows the numbers of cases from 1985 to 31 December 2016. Annual numbers were 8 to 10 p.a. from 2000 to 2003 , after which there was an annual increase from between 15 and 23 p.a. from 2004 to 2009 , rising from 2010 to the current level of between 36 and 65 cases p.a. Estimates of CL wear prevalence are given for datapoints throughout this period.

\section{Case-control study}

There were 63 confirmed AK cases in reusable CLs wearers, with no exclusions: 18 cases, diagnosed up to 22 months before 18 February 2013 (when ethical approval was obtained), completed the study questionnaire retrospectively. The remaining $45 \mathrm{AK}$ cases and all the controls completed the questionnaires at the time of diagnosis. Cases were compared independently with the two different control datasets; one additional case was excluded when the initial clinical diagnosis of AK was not confirmed on follow-up. There were 56 eligible controls from the first control dataset from which CL using controls were excluded if they had CL-related disease. Following the analysis of this dataset, concern was expressed by an external advisor about a potential bias in the selection of these controls. As a result, we reopened the study to obtain the second and fourfold larger control dataset of 213; exclusions were true daily disposable and overnight wear lens users, CL users with AK, 15 with missing CL solution or CL brand information (despite three contact attempts), one who left the hospital before being seen for diagnosis and two whose records could not be retrieved to confirm the outcome. The findings from the analysis of the first dataset were similar to those of the second. The second dataset was chosen for the analysis reported here as giving a more conservative estimate of some ORs. Differences between the two datasets made their combination inappropriate. The results of the assessment of putative risk factors for $\mathrm{AK}$, carried out independently for each exposure without adjustment for confounding, are shown in online supplementary table 4 for the 63 AK cases compared with the entire second control dataset of 213 . This control dataset includes both subsets with $(n=109)$ and without $(n=104)$ CL-related diseases. Both of these subsets of controls were included in the analysis as being likely to give a conservative estimate of the relative risk of exposures for AK, given that these may be shared with the risks for some of the non-Acanthamoeba CL-related keratitis conditions listed in online supplementary table 1 which made up $64 / 109(59 \%)$ of the diagnoses in this subset. The mean age in the whole sample was 33.9 (SD 12.26). The median age was 30 (range 13-76), with 25 th and 75th percentiles of 25-40 (IQR). The distribution was similar in cases and controls: median of 30 in both and IQR 25-49 and 25-38, respectively. Thirty-three of $63(52 \%)$ of the cases and 152/213 (71\%) of the controls were women.

\section{MV analysis for the control dataset of 213 controls}

The results of the analysis for reusable soft CLs, adjusted for confounding, are shown in table 2 . These show significantly increased risks of $\mathrm{AK}$ as a result of the following:

- The use of Oxipol disinfection of 4.74 (CI 1.83 to $12.30, \mathrm{p}$ $0.001)$.

- Wearing group IV (high water content, ionic hydrogel lenses) of 6.71 (CI 1.31 to 34.29 p 0.022 ).

- Poor CL hygiene practice of 3.34 (CI 1.52 to 7.38 p 0.003).

- No, or uncertain, hand washing before lens handling of 3.65 (CI 1.52 to 8.77 p 0.004).

- Wearing CLs in swimming pools or hot tubs of 3.49 (CI 1.51 to $8.04 \mathrm{p} 0.003$ ).

- British (white) ethnicity of 4.82 (CI 1.61 to 14.46 p 0.005 ).

- Occupation other than professional/director/senior official of 3.51 (CI 1.52 to $8.11 \mathrm{p} 0.003$ ).

Rigid gas permeable CL solutions were included in the lens disinfectant analysis, and these solutions were associated with a higher risk of keratitis compared with the referent: this does not imply a higher risk for $\mathrm{AK}$ associated with rigid lens use per se.

\section{DISCUSSION}

The current outbreak of AK in south east England and the UK The incidence study has confirmed a current UK outbreak in south east England starting in 2010/2011, resulting in a mean of 50.3 p.a. (range 36-65) treated at Moorfields for the years 2011-2016 compared with the most accurate estimate of the numbers between outbreaks, of 18.5 p.a. at Moorfields from the prospective national audit carried out over 2 years in $1997-$ 1999. ${ }^{4}$ Given the limitations of the data collection methodology, particularly for the earlier years of the period 2000 to 2012, it is possible that the numbers of cases between 2000 and 2003 are underestimates. On the other hand, the apparent rise in cases in 2004, that was subsequently maintained until a further rise in 2010-2011, may relate to the outbreak in the USA due to the failure of the CL disinfectant AMO Complete Moisture Plus. The latter resulted in a 17-fold rise in cases in the USA but, following withdrawal of the solution, numbers have remained at similar levels for reasons that are unclear. ${ }^{11}$ The industry data on CL user numbers in figure 1 suggest that this current UK outbreak is independent of changes in the prevalence of CL wear. 
Table 1 Distribution of cases and controls for the variables that were potential risk factors for AK chosen for inclusion in the MV analyses

\begin{tabular}{|c|c|c|c|c|c|c|}
\hline Variable & Control & Case & Total & $\mathrm{OR}^{*}$ & $P$ values & $95 \% \mathrm{Cl}$ for $\mathrm{OR}$ \\
\hline \multicolumn{7}{|l|}{ Lens disinfectant: classified by the principal active ingredient (PAI) $\dagger$} \\
\hline 1: Polyhexanide (0.00 005-0.0001) & 90 & 11 & 101 & Referent & & \\
\hline 2: PQ+ALDOX/PQ+Alex $\ddagger$ & 26 & 9 & 35 & 2.83 & 0.038 & 1.06 to 7.57 \\
\hline 3: Hydrogen peroxide $3 \%$ & 12 & 5 & 17 & 3.41 & 0.048 & 1.01 to 11.51 \\
\hline 4: Polyquad-1(0.0001\%)+polyaminopropyl biguanide & 11 & 2 & 13 & 1.49 & 0.633 & 0.29 to 7.60 \\
\hline 5: Oxipol & 55 & 29 & 84 & 4.31 & $<0.001$ & 2.00 to 9.33 \\
\hline 6: Rigid gas permeable lens solution & 11 & 5 & 16 & 3.72 & 0.036 & 1.09 to 12.71 \\
\hline Unknown & 8 & 2 & 10 & & & \\
\hline Total & 213 & 63 & 276 & & & \\
\hline \multicolumn{7}{|l|}{ CL materials classification: simplified from the ANSI classification§ } \\
\hline Groups I+II+III & 18 & 6 & 24 & 1.95 & 0.233 & 0.65 to 5.83 \\
\hline Group IV & 13 & 7 & 20 & 3.15 & 0.039 & 1.06 to 9.37 \\
\hline Group VA & 76 & 13 & 89 & Referent & & \\
\hline Groups VB+VC & 73 & 29 & 102 & 2.32 & 0.023 & 1.12 to 4.81 \\
\hline Rigid gas permeable materials & 11 & 5 & 16 & 2.66 & 0.113 & 0.79 to 8.91 \\
\hline Unknown & 22 & 3 & 25 & & & \\
\hline Total & 213 & 63 & 276 & & & \\
\hline \multicolumn{7}{|l|}{ Hygiene score: categories split between the top (worst) quartile of the sample and the rest } \\
\hline 1: Good-moderate (score $1.75-5.08$ ) & 174 & 30 & 204 & Referent & & \\
\hline 2: Poor (score 5.09-8.08) & 39 & 33 & 72 & 4.91 & $<0.001$ & 2.68 to 8.98 \\
\hline Total & 213 & 63 & 276 & & & \\
\hline \multicolumn{7}{|l|}{ Hand washing before handling CLs } \\
\hline 1: No/unsure & 27 & 21 & 48 & 3.71 & $<0.001$ & 1.90 to 7.22 \\
\hline 2: Yes & 186 & 39 & 225 & Referent & & \\
\hline Unknown & 0 & 3 & 3 & & & \\
\hline Total & 213 & 63 & 276 & & & \\
\hline \multicolumn{7}{|l|}{ Showering when wearing $\mathrm{CLs}$} \\
\hline 1: No & 141 & 25 & 166 & Referent & & \\
\hline 2:Yes & 72 & 38 & 110 & 2.98 & $<0.001$ & 1.67 to 5.31 \\
\hline Total & 213 & 63 & 276 & & & \\
\hline \multicolumn{7}{|l|}{ Water activity using CL's: categories combined } \\
\hline 1: None & 114 & 20 & 134 & Referent & & \\
\hline 2: Ocean/sea/river/lake & 42 & 9 & 51 & 1.22 & 0.649 & 0.52 to 2.89 \\
\hline 3: Public or private pool/hot tub & 57 & 31 & 88 & 3.10 & 0.001 & 1.63 to 5.91 \\
\hline Unknown & 0 & 3 & 3 & & & \\
\hline Total & 213 & 63 & 276 & & & \\
\hline \multicolumn{7}{|l|}{ Ethnic group: for categories* * } \\
\hline 4: British (white) & 141 & 54 & 195 & Referent & & \\
\hline 6: Other & 72 & 6 & 78 & 0.22 & 0.001 & 0.09 to 0.53 \\
\hline Unknown & 0 & 3 & 3 & & & \\
\hline Total & 213 & 63 & 276 & & & \\
\hline \multicolumn{7}{|l|}{ Occupation: for categories $\dagger \dagger$} \\
\hline 1-3 Professional/director/manager/associate professional and technical/senior officials & 163 & 36 & 199 & Referent & & \\
\hline 4-9 Combined & 50 & 22 & 72 & 1.99 & 0.029 & 1.07 to 3.70 \\
\hline Unknown & 0 & 5 & 5 & & & \\
\hline Total & 213 & 63 & 276 & & & \\
\hline
\end{tabular}

${ }^{*}$ ORs are not adjusted for confounding effects of other variables.

tClassification of $\mathrm{CL}$ solutions is given in online supplementary table 2 .

$\neq P A I$ category 2: PQ +ALDOX/PQ +Alex=Polyquad-1 $(0.001 \%)+$ ALDOX $(0.0005 \%)$ or Polyquad- $1(0.0003 \%)+$ alexidine $(0.00016 \%)$-the latter for 4 controls and 1 case only.

$\S$ American National Standard Institute (ANSI) standard Z80.20-2016. American national standard for ophthalmics—contact lenses—standard terminology, tolerances, measurements and physicochemical properties (ophthalmic).

१Hygiene scores were calculated for the 14 hygiene variables listed in online supplementary table 5 using the rules described in that table. The mean score for each patient was then used to divide the patient sample into a simple binary classification for MV analysis in which the worst quartile (the quartile with the highest score) of the sample was categorised as having "Poor" hygiene and compared with the rest categorised as having "Good-Moderate" hygiene. "Good-Moderate" mean scores were 1-75-5.08 and "Poor" mean scores were 5.09-8.08. Hand washing before handling

CLs and showering while wearing CLs were kept as separate variables and and analysed as such.

**Ethnic categories (UK census categories): 1: Asian or Asian British (Bangladeshi, Indian, Pakistani); 2:black or black British (African, Caribbean, other); 4: British (white); 6: other.

††Occupation: 1: managers, directors and senior officials; 2: professional occupations; 3: associate professional and technical occupations; 4: administrative and secretarial occupations; 5: skilled trades occupations; 6: caring, leisure and other service occupations; 7: sales and customer service occupations; 8: process, plant and machine operatives; 9 : occupations requiring no specific training or skills and student: categorised by parents' occupation.

AK, Acanthamoeba keratitis; $\mathrm{CL}$, contact lens; MV, multivariate. 
Table 2 Independent riskfactors for AK from multiple logistic regression models using the main analysis sample (213 controls and 63 AK cases). ORs are adjusted for possible confounding

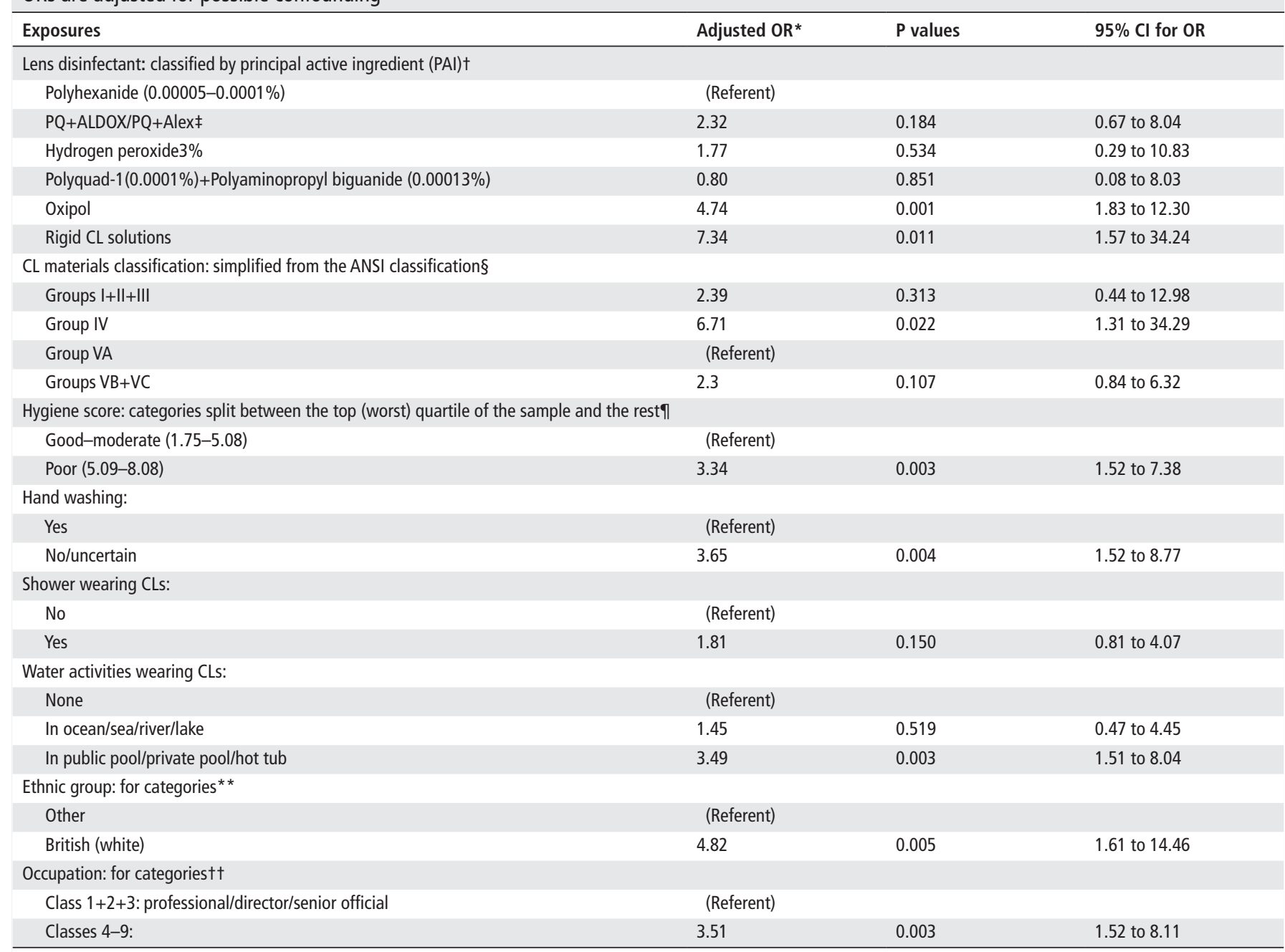

*In constructing each final MV model, one exposure variable was considered as the 'exposure of main interest' and all others as 'auxiliary factors' (potential confounders). The final MV model was thus optimised to estimate the adjusted OR for the exposure of main interest' (see online supplementary table 6 covariate adjustment).

tClassification of $\mathrm{CL}$ solutions is given in online supplementary table 2 .

ҒPAI category PQ+ALDOX/PQ+Alex=Polyquad-1 $(0.001 \%)+$ ALDOX $(0.0005 \%)$ or Polyquad-1 $(0.0003 \%)+$ Alexidine $(0.00016 \%)$-the latter for 4 controls and 1 case only $\S A m e r i c a n$ National Standard Institute (ANSI) standard Z80.20-2016. American national standard for ophthalmics—contact lenses—standard terminology, tolerances, measurements and physicochemical properties (ophthalmic).

१Hygiene scores were calculated for the 14 hygiene variables listed in online supplementary table 5 using the rules described in that table. The mean score for each patient was then used to divide the patient sample into a simple binary classification for MV analysis in which the worst quartile (the quartile with the highest score) of the sample was categorised as having "Poor" hygiene and compared with the rest categorised as having "Good-Moderate" hygiene. "Good-Moderate" mean scores were 1-75-5.08 and "Poor" mean scores were 5.09-8.08. Hand washing before handling CLs and showering while wearing CLs were kept as separate variables and and analysed as such.

**Ethnic categories (UK census categories): 1: Asian or Asian British (Bangladeshi, Indian, Pakistani); 2: black or black British (African, Caribbean, other); 4: British (white); 6 : other.

††Occupation: 1: managers, directors and senior officials; 2: professional occupations; 3: associate professional and technical occupations; 4: administrative and secretarial occupations; 5: skilled trades occupations; 6: caring, leisure and other service occupations; 7: sales and customer service occupations; 8: process, plant and machine operatives; 9 : occupations requiring no specific training or skills and student: categorised by parents' occupation.

AK, Acanthamoeba keratitis; CL, contact lens; MV, multivariate.

\section{Risk factors for AK}

Between $80 \%$ and $90 \%$ of AK cases are potentially avoidable if effective disinfection systems are used, good CL hygiene practice followed and exposure to water while using lenses is avoided. ${ }^{47}$ This current study confirms these previous risk factors and identifies new independent risk factors including deficient hand washing, race and occupation-associated risks (which are probably surrogates for safe CL lens use either from not receiving or following appropriate instruction). It has also confirmed our initial hypothesis that a CL solution may have been associated with the outbreak given that Oxipol-based disinfection was also an independent risk factor (OR 4.74, CI 1.83 to $12 \cdot 30, \mathrm{p} 0.001$ ). This risk is relatively low compared with that of the previous outbreaks of AK associated with CL solution failures: a 40-fold higher risk of AK for users of chlorine-based disinfection systems in the $\mathrm{UK}^{7}$ and 17 -fold higher for users of AMO Complete Moisture Plus in the USA. ${ }^{811}$ The occurrence of AK in an individual patient who has used Oxipol cannot be attributed only to the Oxipol disinfection because: AK develops in some patients who do not use Oxipol (not a necessary cause), and also many patients who use Oxipol do not develop AK (not a sufficient cause). The same applies to the other risk factors. Since the time 
of the investigation Oxipol solution has been phased out by the manufacturer.

Although most manufacturers test their solutions for activity against Acanthamoeba, this is not mandatory, largely because there is no standard methodology for reproducible in vitro sensitivity testing; this has resulted in a wide range of results for different solutions depending on the strains tested, their age and the storage and encystment methodology used. ${ }^{17}$ Independent testing, using rigorous methods, has shown that most multipurpose CL disinfection solutions in vitro may be ineffective against Acanthamoeba. ${ }^{17}$ The issue of mandatory testing of CL disinfection solutions for activity against Acanthamoeba is being actively addressed in the USA ${ }^{17}$, and an international testing standard is being developed by the ISO TC 172/SC 7/WG 9 committee for CL care products. However, disinfection is not sterilisation, and current antimicrobial test standards demand log reductions not elimination. It is also important to understand that CL disinfection solution efficacy is not just related to the principal active ingredients, which is how the analysis in this study was performed, but is the sum of a complex interaction of the disinfectants with the excipients; storage bottle; CL case; debris in the CL case and the CL material. All of these may affect disinfection capability, a topic beyond the scope of this report but relevant to the fact that in this study, we found polyhexanide-based disinfection systems to be the most effective whereas polyhexanide was also the disinfectant in AMO Complete Moisture Plus; the principal cause of the last USA outbreak, but for which the disinfectant failure was attributed to other components. ${ }^{8}$

Another novel independent risk factor identified in this study was the association with the use of group IV CLs (OR 6.71, CI 1.31 to 34.29 , p 0.022). This had been identified as a borderline risk in our study of the previous UK outbreak. ${ }^{7}$ Etafilcon A is one of many group IV materials and is one of the most widely used in CL manufacture. However, in a previous study on the risks of CL-associated keratitis etafilcon A, when used as a daily disposable lens, was found to be less associated with (predominantly bacterial) keratitis than other lens types. ${ }^{14}$ We think that the potential reduction in the risk of the much more common problem of bacterial keratitis is likely to offset a possible increase in the risk of AK associated with group IV CL materials. Therefore, we suggest that group IV lens material users, rather than changing lens material, should optimise their lens hygiene and avoid wearing CLs when exposed to water to minimise their risk of developing AK (which is already very small). Lens cleaning has the capacity to remove adherent Acanthamoeba. ${ }^{18}$ This paradoxical finding for group IV lens materials, associated with potentially higher risks for AK but lower risks for bacterial keratitis, may be explained by differences in adhesion of Acanthamoeba and bacteria to different CL materials. Although not always consistent, and also strain dependent, bacteria generally adhere less to etafilcon A than to hydrophobic lens surfaces (on group III and many group V silicone hydrogels). ${ }^{19}$ Conversely, Acanthamoeba adherence has been shown to be greater to high water content and ionic hydrogel CLs (group IV lenses) compared with low water content hydrogel CLs (groups I and III) ${ }^{20}$ although this may also be strain dependent. ${ }^{18}$ On the other hand, Acanthamoeba adhesion was shown to be higher to first and second generation group V CLs than to etafilcon A (group IV) in another study, which demonstrates some of the limitations of the in vitro investigation of microorganism adherence to unworn CL surfaces given that this difference was reduced by the effects of lens wear deposits and bacterial biofilm (both of which enhance Acanthamoeba trophozoite adhesion). ${ }^{21}$
Research findings that support the risks of exposure to contaminated water are numerous. Deficient hand washing (including drying after washing) and water activities while using CLs have been associated with $\mathrm{AK}$ in this and other studies. Showering in CLs was not a statistically significant independent risk factor in this study but, given known risks of exposure to contaminated water while wearing lenses in other contexts, this should be avoided. Contact lens case contamination by Acanthamoeba spp has been found in 1\%-7\% of asymptomatic CL users. ${ }^{22}$ Most water that CL users are exposed to may be contaminated by Acanthamoeba; domestic tap water in Turkey, ${ }^{23}$ South Korea, ${ }^{24}$ Hong $\mathrm{Kong}^{22}$ and the $\mathrm{UK}^{3}$ as well as swimming pool, hot tub and lake water. ${ }^{25}$ Furthermore, in a UK study, genetically identical organisms were isolated in the domestic water supply of six patients having AK. In the UK the disease is more common in hard water areas, ${ }^{3}$ probably because limescale in taps provides an optimal environment for the organism. ${ }^{4}$ A seasonal association with AK has been related to increased participation in swimming in the summer months. ${ }^{11}$ To establish whether there might have been a 'London Olympic effect' since 2012, resulting from increased participation in swimming by CL users, we compared control data from a 2004/2005 study ${ }^{14}$ with this current data, but have shown no difference in swimming activity amongst CL users for these two periods (online supplementary table 7).

Given the importance of contaminated domestic water supplies in these studies, it follows that small changes in the disinfection of domestic water supplies, that might lead to an increase in the exposure of the population to water contaminated by Acanthamoeba, could have a substantial effect on incidence of AK. The incidence of AK among CL users has historically been 5 to 15 -fold higher in the UK than in other countries, probably as a result of contaminated domestic tank stored tap water. ${ }^{34}$ In the USA implementation of US Environmental Protection Agency legislation in 2002, designed to reduce chlorine generated, potentially carcinogenic, disinfection by-products, was temporally related to an outbreak of AK in the Chicago suburbs. This legislation had resulted in treatment plants introducing a number of measures to minimise disinfection by-products including a reduction in the amount of chlorine used and a switch to the use of chloramine, a less potent disinfectant than chlorine. These measures may have resulted in increased microbial contamination of the water delivery pipes, providing a larger food source for Acanthamoeba and resulting in increased Acanthamoeba contamination at end-user sites. ${ }^{26}$ However, the use of chloraminated water supplies (not implemented and therefore not relevant to the Chicago outbreak) was excluded as a potential cause of the 2004 national outbreak of AK in the USA. ${ }^{8}$ In the UK, the 1998 European Council Directive 98/83/EC shared similar aims. Although the directive was not introduced formally into UK national law until January $2010,{ }^{27}$ coinciding with the start of the current outbreak of AK, informal discussion with three of four major suppliers of water to the south east, and scrutiny of the Drinking Water Inspectorate website, ${ }^{27}$ has not identified any changes in disinfection procedures in 2010-2011 which might have led to increased end-user exposure to contamination by Acanthamoeba, above what are probably historically high levels due to both the widespread use of domestic tank stored water in the UK and the hard water supplies which dominate in south east England.

A potential weakness of this study is the difference in recruitment periods for cases and controls. We do not think that this is likely to have resulted in significant bias, due to changes in exposure to CL solutions, as there was overlap between the recruitment periods for the cases and that for the first control group 
for which findings were confirmed. Unfortunately, changes in market share of CL disinfection solutions are proprietary to the manufacturers who have not felt able to make these available to us. On the other hand, one of the strengths of this study is our proven rationale for the choice of both cases and controls from patients attending Moorfields A\&E service. This minimises potential recruitment bias by recruiting both from a similar catchment area. We have followed this practice in previous studies. ${ }^{71428}$ In the 2008 study, we used community-derived CL controls chosen from the postal code areas in which the cases were living for comparison with the A\&E control population. However, the results of the analyses were no different for the community controls (both time consuming and costly to recruit) compared to those from the controls derived from the A\&E Department. ${ }^{14}$ As a result we chose to use only A\&E Department controls in the current study.

This study has confirmed a persisting outbreak of AK in the south east of England, starting in 2010-2011. This probably reflects the situation elsewhere in the UK, at least for hard water areas. AK should be a largely preventable disease with over $90 \%$ of CL users developing AK having identifiable, avoidable risk factors. ${ }^{4}$ We think it unlikely that the current outbreak results from any of these current risk factors in isolation. However, if the following measures are taken the risk of developing $\mathrm{AK}$ is likely to be very low: improving CL and hand hygiene when inserting lenses, avoiding the contamination of CLs with water including when engaging in water activities while using CLs, avoiding the use of CL solutions by switching to daily disposable CL use or, for those continuing to wear reusable lenses, maintenance of optimal lens care with effective solutions. Publicity for these measures, as in the 1991-1995 UK outbreak, ${ }^{12}$ can be expected to rapidly reduce the incidence of AK. Water avoidance publicity for CL users (by water companies, water sports facilities, the CL industry and eye care professionals) can be expected to reduce the incidence in the longer term. The addition of 'no water' labelling on all CL packaging ${ }^{29}$ should become mandatory. Ongoing surveillance of AK incidence by major UK ophthalmic units will identify future changes in incidence more rapidly than for this current outbreak and trigger the establishment of a case control study to identify avoidable causes. Surveillance of end-user domestic water contamination by the water companies is currently limited to measuring faecal bacterial contamination: the addition of Acanthamoeba to this panel can be expected to contribute to our understanding of local variations in the risk of exposure and make CL users aware of the importance of maintaining preventive measures.

\begin{abstract}
Acknowledgements Moorfields Eye Hospital staff: the Accident \& Emergency Department Nurses for their help in identifying control contact lens users. Melanie Mason and the Corneal Clinic staff for assistance with recruiting cases. Varshini Parayoganathan and Sophie Connor in Research and Development for database management.
\end{abstract}

Contributorship NC: submitted the research and ethics applications, recruited the cases, co-ordinated recruitment of the first set of controls, prepared the incidence dataset from 2000 to 2014 and contributed to the planning and execution of all aspects of the study. JJH: collected the incidence data for 2015/2016. SV: facilitated the identification of controls and cases in the Moorfields A\&E Deptartment. CFR: assisted questionnaire design, the dataset collection and recruited the second set of controls. DCM: contributed to study planning and carried out the statistical analysis. JKGD: planned the study, assisted with the data collection and co-ordinated the preparation of the manuscript. All authors contributed to the manuscript.

Funding Grants from Fight for Sight (1542/43 and 1465/6), Moorfields Eye Hospital Special Trustees (ST 12 09A), anonymous donors to Moorfields Eye Charities, and from CooperVision UK. Part of John Dart's salary was paid by the National Institute of Research (NIHR) Biomedical Research Centre (BRC) at
Moorfields Eye Hospital NIHR BRC. Dr Carnt was supported by an NHMRC Early Career CJ Martin Fellowship (APP1036728).

Disclaimer None of the funders had a role in the design or conduct of this research although one reviewed the manuscript; modifications were limited to clarification and grammatical changes. The views expressed are those of the author(s) and not necessarily those of the NHS, the NIHR or the Department of Health.

Competing interest statement CooperVision UK, one of whose products has been identified by this study as a risk factor contributing to the development of Acanthamoeba keratitis, funded the recruitment of the second set of controls. This was to verify the findings of the first set as there was a perception that the selection of these may have been biased. This did not prove to be the case and the company has since phased out the product. CooperVision did not influence the study design. Representatives of CooperVision reviewed the manuscript but made no changes other than clarifications and grammatical changes.

Patient consent Not required.

Ethics approval National Research Ethics Service Committee London-Hampstead, REC Reference 13/LO/0032 and the Moorfields Eye Hospital Research Governance Committee.

Provenance and peer review Not commissioned; externally peer reviewed.

Data sharing statement None are available. The study database, from which the reported analysis has been done, can be provided if this is requested by a commercial organisation who wishes to contest the findings. We believe we have precluded any real likelihood of such a request being made by having had extensive pre-submission discussions with CooperVision, whose product is identified as being associated with an increased risk of AK. They have agreed the contents of the manuscript.

\section{REFERENCES}

1 Dart JK, Saw VP, Kilvington S. Acanthamoeba keratitis: diagnosis and treatment update 2009. Am J Ophthalmol 2009:148:487-99.

2 Carnt N, Robaei D, Minassian A. Acanthamoeba keratitis in 194 patients: risk factors for poor outcomes and severe inflammatory complications. Br J Ophthalmol 2017:pii: bjophthalmol-2017-310806.

3 Kilvington S, Gray T, Dart J, et al. Acanthamoeba keratitis: the role of domestic tap water contamination in the United Kingdom. Invest Ophthalmol Vis Sci 2004;45:165-9.

4 Radford CF, Minassian DC, Dart JK. Acanthamoeba keratitis in England and Wales: incidence, outcome, and risk factors. Br J Ophthalmol 2002;86:536-42.

5 Stehr-Green JK, Bailey TM, Brandt FH, et al. Acanthamoeba keratitis in soft contact lens wearers. A case-control study. JAMA. 1987;258:57-60.

6 Cope JR, Collier SA, Schein OD. Acanthamoeba keratitis among rigid gas permeable Contact Lens Wearers in the United States, 2005 through 2011. Ophthalmology 2016;123:1435-41.

7 Radford CF, Bacon AS, Dart JK, et al. Risk factors for acanthamoeba keratitis in contact lens users: a case-control study. BMJ 1995;310:1567-70.

8 Verani JR, Lorick SA, AcanthamoebaKeratitis Investigation Team. National outbreak of Acanthamoeba keratitis associated with use of a contact lens solution, United States. Emerg Infect Dis 2009;15:1236-42.

9 Jasim H, Knox-Cartwright N, Cook S, et al. Increase in acanthamoeba keratitis may be associated with use of multipurpose contact lens solution. BMJ 2012;344:e1246.

10 Chawla A, Armstrong M, Carley F. Acanthamoeba keratitis - an increasing incidence. Cont Lens Anterior Eye 2014;37:120.

11 Yoder JS, Verani J, Heidman N, et al. Acanthamoeba keratitis: the persistence of cases following a multistate outbreak. Ophthalmic Epidemiol 2012;19:221-5.

12 Morlet N, Duguid G, Radford C, et al. Incidence of acanthamoeba keratitis associated with contact lens wear. Lancet 1997;350:414.

13 Robaei D, Carnt N, Minassian DC, et al. The impact of topical corticosteroid use before diagnosis on the outcome of Acanthamoeba keratitis. Ophthalmology 2014:121:1383-8.

14 Dart JK, Radford CF, Minassian D, et al. Risk factors for microbial keratitis with contemporary contact lenses: a case-control study. Ophthalmology 2008;115:1647-54.

15 Radford CF, Minassian D, Dart JK, et al. Risk factors for nonulcerative contact lens complications in an ophthalmic accident and emergency department: a case-contro study. Ophthalmology 2009;116:385-92.

16 Stapleton F, Dart J, Minassian D. Nonulcerative complications of contact lens wear. Relative risks for different lens types. Arch Ophthalmol. 1992;110:1601-6.

17 Johnston SP, Sriram R, Qvarnstrom Y, et al. Resistance of Acanthamoeba cysts to disinfection in multiple contact lens solutions. J Clin Microbiol 2009;47:2040-5.

18 Kilvington S. Acanthamoeba trophozoite and cyst adherence to four types of soft contact lens and removal by cleaning agents. Eye 1993;7(Pt 4):535-8.

19 Kodjikian L, Casoli-Bergeron E, Malet F, et al. Bacterial adhesion to conventional hydrogel and new silicone-hydrogel contact lens materials. Graefes Arch Clin Exp Ophthalmol 2008;246:267-73. 
20 Lema I, Rodríguez-Ares MT, Gómez-Torreiro M, et al. Adherence of Acanthamoeba to unworn conventional and disposable soft contact lenses. Cornea 2001;20:635-8.

21 Beattie TK, Tomlinson A, McFadyen AK. Attachment of Acanthamoeba to first- and second-generation silicone hydrogel contact lenses. Ophthalmology 2006;113:117-25.

22 Boost M, Cho P, Lai S. Detection of acanthamoeba in tap water and contact lens cases using polymerase chain reaction 11. OptomVisSci 2008:85:526-30.

23 Koltas IS, Eroglu F, Erdem E, et al. The role of domestic tap water on Acanthamoeba keratitis in non-contact lens wearers and validation of laboratory methods. Parasitol Res 2015;114:3283-9.

24 Jeong HJ, Yu HS. The role of domestic tap water in Acanthamoeba contamination in contact lens storage cases in Korea. Korean J Parasitol 2005;43:47-50.
25 Niyyati M, Saberi R, Latifi A, et al. Distribution of acanthamoeba genotypes isolated from recreational and therapeutic geothermal water sources in southwestern iran. Environ Health Insights 2016;10:EHI.S38349-74.

26 Joslin CE, Tu EY, McMahon TT, et al. Epidemiological characteristics of a Chicago-area Acanthamoeba keratitis outbreak. Am J Ophthalmol 2006;142:212-7.

27 Water CloD, 2011. Drinking water 2011 London and South East region of England. PublicWater Supplies Regional Reports. Available from: http://www.dwi.gov.uk/about/ annual-report/2011/london\&se.pdf [Accessed 24 Dec 2017].

28 Dart JK, Stapleton F, Minassian D. Contact lenses and other risk factors in microbial keratitis. Lancet 1991;338:650-3.

29 McCormick E, Derbyshire L, 2017. Campaigning recognition for "no water" campaign 2016. Available from: https:/www.aop.org.uk/ot/industry/charity/2016/06/13/ campaigning-recognition [Accessed 23 Dec 2017]. 